\title{
Ascites as a sole presentation of juvenile granulosa cell tumor
}

\begin{abstract}
Granulosa cell tumors (GCT) are rare tumor and they constitute only $2 \%$ of all ovarian tumor. They are categorized in to juvenile and adult types. Juvenile Granulosa cell tumors only contribute to $5 \%$ of all GCT and they usually affect young children and premenarchal girls. These tumor are mainly hormone producing and mostly present with signs of precocious puberty. However other clinical presentation like acute abdomen or abdominal distention has also been reported. Here we present a 2 years old girl who reported to us with complaints of progressive abdominal distention and urinary retention. Her tumor markers were negative and her ultrasound and $\mathrm{CT}$ with contrast showed a massive abdominal ascites and complex ovarian mass. Exploration and left salpingo-opherctomy with ascitic fluid sampling done. Her ascitic fluid was negative for tumor cell. To our knowledge it is a unique case in which stage $1 \mathrm{~A}$ co-existed with ascites fluid negative of tumor cells.
\end{abstract}

Keywords: granulosa cell tumor, ovarian tumor, pediatric, ascites, juvenile
Volume 3 Issue 2 - 2015

\author{
Manal Dhaiban,' Nadeem Akhtar,' ${ }^{2}$ Maryam \\ Asif, ${ }^{3}$ Ashok Tanwani ${ }^{4}$ \\ 'Pediatric surgery department, Pakistan institute of medical \\ sciences (PIMS), Pakistan \\ ${ }^{2}$ Department of Pediatric surgery, PIMS, Pakistan \\ ${ }^{3}$ Shifa College of medicine, Pakistan \\ ${ }^{4}$ Department of pathology, PIMS, Pakistan
}

\begin{abstract}
Correspondence: Manal Dhaiban, 3rd year resident of M.S pediatric surgery, Shaheed Zul-fiqar Ali Bhutto Medical university, Children hospital/Pakistan Institute of Medical sciences, 437, street \#17, Gali \#76, Sector G-8/I, Islamabad/ Pakistan, Tel 0092-3368819191,

Email manaldhaiban1985@gmail.com
\end{abstract}

Received: October 20,2015 | Published: November 13,2015
Abbreviations: JGCT, juvenile granulosa cell tumor; AFP, alpha feto protein; B.HCG, beta human chorionic gonadotropin; EMA, epithelial membrane antigen; FIGO, International federation of gynecology and obstetrics; H\&E, heamatoxylin and eosin stain

\section{Introduction}

Ovarian granulosa cell tumor constitutes $2 \%$ of ovarian tumors. They are classified into Juvenile (5\%) and Adult type (95\%) based on histological-pathological features and biological behavior. ${ }^{2}$

These are generally hormone producing neoplasm and $80 \%$ of cases present is isosexual pseudoprecocity. ${ }^{3}$ They usually affect young children and pre-menarchal girls. ${ }^{4}$ The diagnosis of JGCT is based on clinical, histo-pathological and immunohistochemistry. ${ }^{3}$

Prognosis mainly depends on the surgical stage. ${ }^{5}$ In majority of early stage disease (FIGO stage 1A) surgery is sufficient; however adjuvant chemotherapy is required for advanced disease. ${ }^{4}$ Here we report an interesting case of JGCT of 2 years old girl who presented with urinary retention and abdominal ascites.

\section{Case report}

A two years old girl presented with history of urinary retention since 2 months. It was associated with decrease urinary output and painful micturation since 1 month. She also had a history of gradual abdominal distention with mild abdominal pain. She is a product of consanguineous marriage with death 2 of her preceding sibling due to unknown reasons at age of 3 months and 2 years. Her general physical examination was normal. Her abdominal examination showed massive abdominal distention with positive fluid thrill and shifting dullness. A rounded, fixed mass felt at the pelvic area with smooth surface. Her rectal examination showed tenderness at the anterior rectal wall. She had normal breast and genetalia. Normal blood \& urine tests. Abdominal ultrasound showed a mixed echogenicity mass measuring $11.5 \times 6.7 \mathrm{~cm}$ with both solid and cystic components arising from the pelvic area with gross ascites. Contrast enhanced CT scan shows massive ascites and a large, heterogenous, solid cystic mass in lower abdomen \& false pelvis $(11 \times 8.1 \times 7.5 \mathrm{~cm})$; originating from the left adenxa with no internal calcification. There was associated with abdomen-pelvic ascites. Rest of abdominal viscera, peritoneium and lymph nodes were unremarkable. Her Alfha fetoprotein and bHCG were normal. On exploratory Laprotomy 1 liter of Ascitic fluid was drained with samples sent for analysis. Left salpingo-opherctomy was done as the mass was originating from the left ovary Figure 1. Uterus, right ovary and lymph nodes were grossly normal. Peritoneal fluid cytology was negative for malignant cells. Grossly the mass was having mixed cystic and solid areas, weighting $1.3 \mathrm{~kg}$ with some focal areas of hemorrhage Figure 2. Histopathological examination Microscopic features show diffuse sheets and macrofollicular pattern of tumor cells. The malignant cells were polygonal and small. The cytoplasm was abundant and amphophilic. The nuclei were atypical without grooves and show conspicuous nucleoli at places. Mitotic activity was more than 10 cells/10 hpf. Large tumor cells with extensive lutenization were seen focally, presenting the cal component of ovarian stroma that is compatible with Juvenile granulosa cell tumor Figure 3. Immuno-histo-chemistry was positive for Inhibin and CD99 and negative for EMA, CD117 and alpha feto-protein. Bone scan and chest radiographs were unremarkable. Based on FIGO staging system this patient is stage IA. On 3 months follow up patient is doing well and her urinary retention relieved, however she is on long term follow up due to late recurrence.

\section{Discussion}

\section{Mass}

Juvenile Granulosa cell tumor contributes to $5 \%$ of all the granulosa cell tumor. They usually occur in young children and premenarchal girls. $^{2}$

JGCT mostly limited to one ovary and bilateralism only occurs in $3 \%$ of cases. ${ }^{6}$ They have natural slow growing tendency thus have very late recurrence as late as 40 years after initial diagnosis. 


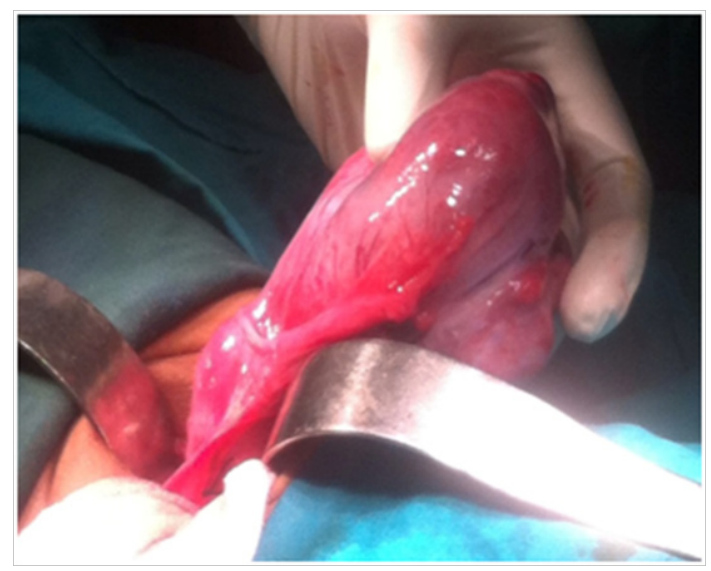

Figure I Intra-operative picture showing the mass originating from the left ovary with intact capsule.

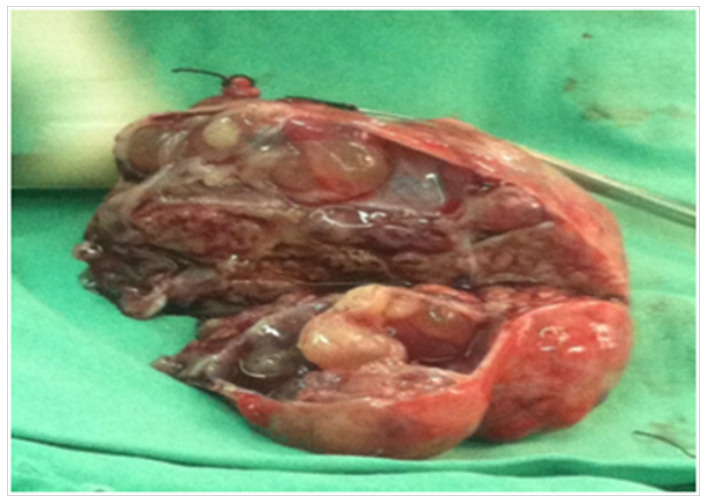

Figure 2 Gross picture of the tumor showing solid and cystic structures.

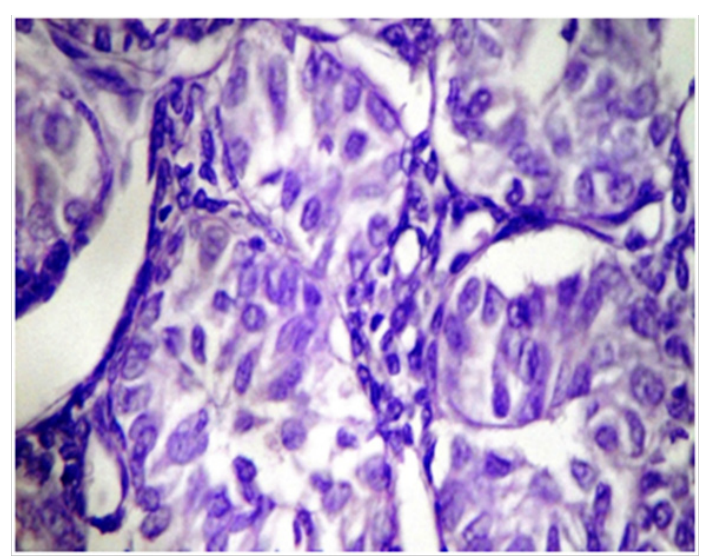

Figure $3 \mathrm{H}$ \& E stain on Light Microscopy (400 magnification) of JGCT show macrofollicles of tumor cells. Neoplastic cells are polygonal with abundant amphophilic cytoplasm. The nuclei are without grooves and may contain conspicuous nucleoli.

\section{Uterus}

These neoplasm are mostly hormone active and usually produce estrogen. The commonest presentation related to the hormone effects is precocious pseudopuberty. ${ }^{7}$ Other presentation is acute abdomen due to tumor rupture and abdominal distention. ${ }^{3}$

Ascites as an associated feature is only present in $10 \%$ of cases. ${ }^{8}$ It is usually in association with advanced disease or tumor rupture, however in our patient the ascitic fluid was negative for tumor cells. To the best of our knowledge this is the 1st case in literature in which ascites was associated with JGCT stage 1A. Ashnagar A et al. reported massive ascites as the main presenting features in 13 year old girl with tumor stage $1 \mathrm{C} .{ }^{3}$

As compared to adult type these tumor show benign biological behavior despite the aggressive histological features such as high mitotic figure and nuclear atypia. ${ }^{8}$

The diagnosis is rarely done be preoperatively especially when there is no signs of precocious pseudopuberty. The specific marker for JGCT is serum inhibin (which is produced by granulosa cells), however other tumor markers like serum CA-125, AFP, Beta HCG has to be measured to rule out ovarian germ cell tumor and epithelial ovarian carcinomas. ${ }^{9}$

The final diagnosis of JGCT is done after Histopathological examination and immunohistochemical panel. JGCT is positive for CD 99 and alpha inhibin and negative for EMA and AFP. ${ }^{6}$ Cases of JGCT with negative inhibin, Calretinin can be of a diagnostic value. ${ }^{1}$

The management of JGCT basically depends on the age of the patient and stage of the disease. ${ }^{4}$ Surgery is the primary initial treatment in all cases. Unilateral salpingo-oopherectomy is the standard treatment of stage 1A tumor. Adjuvant chemotherapy is only required for advanced disease. ${ }^{2}$ Generally the prognosis of stage $1 \mathrm{~A}$ is good and reported 5 year survival rate is up to $90 \% .^{3}$ Long term regular follow up is mandatory in these patient due to late recurrence. ${ }^{9}$

In conclusion, JGCT is a rare entity in children and our case is of interest as it is the 1st case in which stage 1A tumor has co-existed with massive ascites in a 2 years old girl.

\section{Acknowledgments}

None.

\section{Conflicts of interest}

The authors declare there is no conflict of interests.

\section{Funding}

None.

\section{References}

1. Dogra S, Yadav YK, Sharma U, et al. Juvenile granulosa cell tumor with an unusual immunoprofile, presenting as precocious puberty. South Asian Journal of Cancer. 2013;2(3):150.

2. Fink D, Kubik-Huch R, Wildermuth S. Juvenile granulosa cell tumor. Abdom Imaging. 2001;26(5):550-552.

3. Ashnagar A, Alavi S, Nilipour Y, et al. Massive ascites as the only sign of ovarian juvenile granulosa cell tumor in an adolescent: a case report and a review of the literature. Case Rep Oncol Med. 2013:386725.

4. Karalok A, Tasci T, Ureyen I, et al. Juvenile granulosa cell ovarian tumor:clinicopathological evaluation of ten patients. J Turk Ger Gynecol Assoc. 2015;16(1):32-34.

5. Young R, Dickersin G, Scully R. Juvenile granulosa cell tumor of the ovary. A clinicopathological analysis of 125 cases. Am J Surg Pathol. 1984;8(8):575-596.

6. Pectasides D, Pectasides E, Psyrri A. Granulosa cell tumor of the ovary. Cancer Treat Rev. 2008;34(1):1-12. 
7. Fleming N, de Nanassy J, Lawrence S, et al. Juvenile Granulosa and Theca Cell Tumor of the Ovary as a Rare Cause of Precocious Puberty:Case Report and Review of Literature. J Pediatr Adolesc Gynecol. 2010;23(4):e127-e131.

8. Bouffet E, Basset T, Chetail N, et al. Juvenile granulosa cell tumor of the ovary in infants:A clinicopathologic study of three cases and review of the literature. J Pediatr Surg. 1993;32(5):762-765.
9. Sekkate S, Kairouani M, Serji B, et al. Ovarian granulosa cell tumors:a retrospective study of 27 cases and a review of the literature. World $J$ Surg Oncol. 11:142. 